\title{
EVOLUCIÓN DE LA CALIDAD DE SEMILLA DE Capsicum annuum L. DURANTE SU DESARROLLO EN EL FRUTO
}

\section{SEED QUALITY EVOLUTION OF Capsicum annuum L. THROUGH DIFFERENT FRUIT DEVELOPMENT STAGES}

\author{
Misael J. Ayala-Villegas ${ }^{1}$, Óscar J. Ayala-Garay ${ }^{2 \star}$, Víctor H. Aguilar-Rincón ${ }^{2}$ y Tarsicio Corona-Torres ${ }^{2}$
}

\begin{abstract}
${ }^{1}$ DuPont Pioneer NRSP-Puerto Vallarta. Camino Viejo a Valle de Banderas. Km 3 No. 19. 63733, Santa Rosa Tapachula, Nayarit, México. ${ }^{2}$ Postgrado en Recursos Genéticos y Productividad, Colegio de Postgraduados. Km 36.5 Carretera México-Texcoco. 56230, Texcoco, Edo. de México. México.
\end{abstract}

${ }^{*}$ Autor para correspondencia (oayala@colpos.mx)

\section{RESUMEN}

El uso de semillas de calidad representa el inicio de un cultivo rentable. Este estudio se hizo para identificar la etapa de cosecha más apropiada y obtener la mejor calidad de semilla. En Texcoco, México, en condiciones de invernadero se estudió la evolución de la calidad física y fisiológica de semillas de tres tipos de chile: de Árbol, Ancho y Guajillo, obtenidas de frutos cosechados en cinco etapas de desarrollo: (E1) 25 y (E2) 40 d después de antesis, (E3) cuando el fruto comenzó a cambiar de color, (E4) cuando el fruto cambió totalmente de color, y (E5) cuando el fruto estaba totalmente seco. También se evaluaron dos etapas de extracción de semilla: inmediatamente después de la cosecha de fruto (EI) y 15 d después (EP). El contenido de humedad de la semilla fue $7.6 \%$ en todos los tratamientos. Las variables de calidad física (peso de 1000 semillas y el peso volumétrico) y fisiológica (germinación y velocidad de germinación) siguieron una tendencia de incremento sigmoidal, cuya fase de rápido crecimiento fue hasta E3 para la calidad física y hasta E4 para la calidad fisiológica. La extracción en EP mejoró significativamente la calidad fisiológica, pues la semilla de frutos cosechados en E3 alcanzó un promedio de $\mathbf{9 3 . 4} \%$ de germinación, muy superior a la obtenida en las semillas de frutos cosechados en EI (6.7\%). La mejor calidad fisiológica de semillas en los chiles Ancho y de Árbol se alcanzó en frutos cosechados en E4 combinada con extracción EP, mientras que en Guajillo la mejor combinación fue E3 y EP.

Palabras clave: Capsicum annuum, calidad de semilla, etapa de desarrollo, etapa de extracción, germinación.

\section{SUMMARY}

High quality seeds are required in commercial farming to ensure profits. In this research the physical and physiological quality of seeds of Capsicum annuum cvs. de Árbol, Ancho, and Guajillo, was evaluated under greenhouse conditions in Texcoco (México), trough different fruit development stages, to identify the harvest stage with the best seed quality. Seeds were obtained from fruits harvested at five development stages: (S1) 25 and (S2) $40 \mathrm{~d}$ after anthesis; (S3) when fruit started to change color; (S4) when fruit changed completely of color; and (S5) when fruit was fully dried. In addition, seeds were extracted at two postharvest dates: immediately (IE) and $15 \mathrm{~d}$ (PE) after fruits were harvesting in each stage. Seed moisture content was $7.6 \%$ in all treatments. The physical (weight of 1000 seeds, volumetric weight) and physiological (germination, vigor) quality variables of the three cvs. of chilli followed a sigmoidal growth curve, and reached their maximum values when extracted from fruits at the S3 stage for the physical quality, and from fruits harvested at the $\mathrm{S} 5$ stage for the physiological quality. Seed germination occurred earlier when the seeds were extracted at PE than when extracted at IE, since extraction at PE from fruits harvested at the $\mathrm{S} 3$ stage rendered a germination rate of $93.4 \%$, much higher than the $6.7 \%$ rate obtained with seeds extracted from fruits harvested at the EI stage. The best physiological quality was obtained from seeds extracted at PE from fruits harvested at the S4 stage for cvs. Ancho and Chile de Árbol, while for cv. Guajillo the best fruit stage was S3.

Index words: Capsicum annuum, seed quality, development stage, extraction stage, germination.

\section{INTRODUCCIÓN}

Para la producción de un cultivo rentable es necesario utilizar semilla de alta calidad, porque ofrece mayor probabilidad de éxito durante el establecimiento de la plántula. Además, las semillas de alta calidad mantienen mayor viabilidad durante el almacenamiento (Pittcock, 2008). La calidad de semilla comprende aspectos genéticos, fitosanitarios, físicos y fisiológicos. Entre estos últimos se incluyen la viabilidad, la capacidad germinativa y el vigor, que se ven afectados por las condiciones de crecimiento de la planta madre durante el desarrollo de la semilla, el grado de madurez de ésta al momento de la cosecha, la forma de cosechar y las condiciones de beneficio (Pittcock, 2008).

El desarrollo de las semillas se puede dividir en tres fases: histodiferenciación, acumulación de reservas y adquisición de tolerancia a la desecación (Taiz y Zeiger, 2010). La madurez fisiológica es la etapa del desarrollo de la semilla en la que alcanza su máximo peso seco, que corresponde con el fin del periodo de acumulación, y también es cuando ocurre la máxima germinación y vigor. A partir de esta etapa comienza el deterioro de las semillas. En algunas especies la madurez de la semilla coincide con la madurez del fruto, de modo que los frutos cosechados tempranamente proporcionan semillas de baja calidad (Bewley et al., 2013). 
El chile (Capsicum sp.) se ha cultivado desde tiempos precolombinos como parte de la dieta de varias culturas americanas (Long-Solís, 1986). En México, este fruto es una de las hortalizas más importantes debido al consumo popular y la magnitud de la producción. Existen cinco especies cultivadas de chile (Nuez et al., 2003). De éstas, por la extensión de su cultivo y el valor económico que representa su producción, C. annuum L. es la más importante y en México es donde se encuentra la mayor diversidad (Aguilar-Rincón et al., 2010), representada en orden de importancia por los tipos: Serrano, Jalapeño, Ancho, Pasilla, Bell, Guajillo, de Árbol, entre otros (SIAP, 2011), los cuales contrastan en forma, color, olor, sabor y picor de los frutos.

Según Edwards y Sundstrom (1987), entre la madurez del fruto del chile tipo Bell y la de la semilla hay correlación, de manera que la alta calidad de ésta se obtiene cuando el fruto ha cambiado totalmente de color. En pimiento, Valdés et al. (1992) obtuvieron los más altos porcentajes de germinación en semillas extraídas de frutos cosechados completamente rojos (maduros). Por su parte, Randle y Honma (1981) indicaron que las semillas de chile completan su madurez fisiológica una vez que los frutos cosechados pasaron por un periodo de reposo de una a seis semanas, en función del tipo de chile. Se considera entonces que el grado de madurez de la semilla al momento de la cosecha y el tiempo de reposo poscosecha del fruto afectan la calidad, como lo reportaron Vidigal et al. (2009).

De manera empírica se sabe que en chiles de los tipos Serrano y Ancho es posible obtener semillas viables de frutos en estado inmaduro (próximos a cambio de color); no obstante, la extracción generalmente se realiza hasta que el fruto está seco. La extracción de la semilla después de que los frutos maduran, es decir, después de dejarlos en el campo hasta su secado o de colocarlos en cámaras de deshidratación, requiere de tiempo e infraestructura, y además la semilla queda expuesta a factores adversos que pudieran causar la pérdida de su calidad.

La posibilidad de obtener semillas de alta calidad en menor tiempo puede ser útil en programas de mejoramiento genético y conservación de germoplama; en el primer aspecto, la utilización de semilla extraída de frutos "inmaduros" aceleraría el ciclo de selección y permitiría obtener germoplasma mejorado en periodos más cortos; en tanto que para la conservación, una semilla de alta calidad conserva durante más tiempo su poder germinativo y resiste condiciones adversas de almacenamiento.

Los objetivos del presente estudio fueron evaluar las características de calidad de semillas de tres tipos de chile cosechados en cinco etapas de desarrollo del fruto, con extracción inmediata y 15 d después de la cosecha, y con ello determinar en cada tipo la etapa más apropiada para obtener semillas con mayor calidad.

\section{MATERIALES Y MÉTODOS}

\section{Material vegetal}

Se emplearon poblaciones de chile obtenidas del banco de germoplasma del Colegio de Postgraduados, México. Correspondieron a tres tipos de chile: Ancho (CP-667) colectado en 2005 en San Martín Texmelucan, Puebla; de Árbol (CP-1037) obtenido en 2007 de Yahualica, Jalisco; y Guajillo (CP-1112) originario de Ojo Caliente, Zacatecas (2009). Una prueba de germinación previa permitió constatar que las semillas de las tres poblaciones utilizadas tenían al menos $85 \%$ de germinación, lo que muestra que las diferencias en tiempo de almacenamiento después de la cosecha no tuvo efecto importante en esta variable.

\section{Siembra y manejo agronómico}

En Montecillo, Municipio de Texcoco, Edo. de México, ubicado a $98^{\circ} 53^{\prime} \mathrm{LN}, 19^{\circ} 29^{\prime} \mathrm{LO}$ y $2250 \mathrm{~m}$ de altitud, en un invernadero con cubierta de polietileno se sembraron 60 semillas de cada tipo de chile, en charolas de unicel de 200 cavidades rellenadas con sustrato estéril (turba), el 25 de marzo del 2010. Dos semanas después de la emergencia se fertilizó con Fertiguano ${ }^{\circledR}$ (fertilizante líquido constituido de minerales básicos como $\mathrm{N}, \mathrm{P}$ y K, más una mezcla enzimática de ácidos policarboxílicos, oligosacáridos, polifenoles y microorganismos, entre otros) agregada al agua de riego $\left(1 \mathrm{~mL} \mathrm{~L}^{-1}\right)$ y aplicado dos veces por semana hasta el trasplante. El trasplante se realizó el 25 de mayo en bolsas de polietileno negro de $30 \times 30 \mathrm{~cm}$ rellenas con un sustrato compuesto de tierra negra, hojarasca y turba en proporción $2: 1: 1$. En esta fase se aplicaron tres riegos cada semana y dos fertilizaciones con Ultrasol ${ }^{\circledR}$ multipropósito (18N-18P$18 \mathrm{~K}+$ micronutrimentos) aplicado al sustrato junto con el agua de riego $\left(2 \mathrm{~g} \mathrm{~L}^{-1}\right)$. Después se hicieron aspersiones foliares quincenales de dos fertilizantes foliares, primero con Bayfolán Forte ${ }^{\circledR}(11.4 \%$ de N, $8 \%$ de P, $6 \%$ de K + micronutrimentos; $\left.2 \mathrm{~mL} \mathrm{~L}^{-1}\right)$, y desde el inicio de la floración se asperjó NutriCalcio ${ }^{\circledR}\left(8.1 \%\right.$ de N, $12.75 \%$ de Ca; $\left.2 \mathrm{~mL} \mathrm{~L}^{-1}\right)$.

\section{Obtención de la semilla para evaluación y tratamientos}

A partir del momento en que $50 \%$ de las plantas de cada tipo de chile tenían al menos una flor con anteras expuestas, diariamente al medio día se etiquetaron las flores que estuvieran en antesis. Posteriormente se cosecharon 30 frutos de cada tipo en cada una de las siguientes cinco etapas: (E1) 25 y (E2) 40 d después de antesis (dda); (E3) cuando 80 $\%$ de los frutos presentes comenzaban a cambiar de color verde a rojo (60 dda para chile Ancho y Árbol, y 70 dda 
para Guajillo); (E4) cuando 80 \% de los frutos cambiaron totalmente de color (75 dda para los tipos Ancho y Árbol, y 90 dda para Guajillo); y (E5) cuando $80 \%$ de los frutos estuvieron totalmente secos (106 dda para Ancho y Árbol, y 120 dda para Guajillo). Cabe señalar que $5 \mathrm{~d}$ antes de la etapa $\mathrm{E} 5$ ocurrió una helada $\left(-3^{\circ} \mathrm{C}\right)$. A 15 de los frutos recolectados se les extrajo la semilla inmediatamente (EI), y en el resto de frutos la extracción de semilla se hizo $15 \mathrm{~d}$ después de ser almacenados a $18 \pm 1{ }^{\circ} \mathrm{C}(\mathrm{EP})$.

La extracción de la semilla se hizo en forma manual. Las semillas se lavaron con agua corriente durante $1 \mathrm{~min}$ y se dejaron secar sobre toallas de papel durante $24 \mathrm{~h}$ a $20^{\circ} \mathrm{C}$, luego se colocaron en sobres de papel y se almacenaron 2 meses a $18 \pm 2{ }^{\circ} \mathrm{C}$.

\section{Variables de respuesta}

Contenido de humedad (CH, \%). Una muestra de $0.5 \mathrm{~g}$ de semilla se secó en estufa a $103^{\circ} \mathrm{C}$ durante $17 \pm 1 \mathrm{~h}$ para obtener su peso seco (final), que junto con el peso inicial de la muestra se usó para calcular el contenido de humedad del lote de semillas (ISTA, 2004).

Peso de 1000 semillas (PS, g). Se midió en cuatro repeticiones de 50 semillas, con una balanza OHAUS ${ }^{\circledR}$ modelo Adventurer (Beijing, China), y con el dato se calculó el peso correspondiente a 1000 semillas (ISTA, 2004).

Peso volumétrico (PV, $\left.\mathrm{kg} \mathrm{hL}^{-1}\right)$. En una probeta de $5 \mathrm{~mL}$ se midió el volumen de $0.5 \mathrm{~g}$ de semilla y se pesó en una balanza OHAUS ${ }^{\circledR}$ modelo 300 (Beijing, China) en cuatro repeticiones, y luego se hizo la conversión a las unidades correspondientes (ISTA, 2004).

Germinación (PG, \%) y velocidad de germinación (VG, radículas $\left.\mathrm{d}^{-1}\right)$. La prueba se condujo en un cuarto de germinación a $25 \pm 1{ }^{\circ} \mathrm{C}$, con luz constante. Se emplearon cuatro repeticiones de 25 semillas tratadas con fungicida Captán ${ }^{\circledR}$ (N-(triclorometiltio)ciclohex-4-en-1,2-dicarboximida) a 2 $\mathrm{g} \mathrm{L}^{1}$, que fueron colocadas en cajas Petri sobre una capa de papel filtro Whatman No. 2 humedecido con $4.5 \mathrm{~mL}$ de $\mathrm{KNO}_{3}$ a $2 \%$. Diariamente se contabilizaron las semillas germinadas (con protrusión de la radícula) hasta los $21 \mathrm{~d}$, lo que permitió obtener la cinética de la germinación. Con el total de semillas germinadas se calculó el porcentaje de germinación (PG). La velocidad de germinación (VG) se calculó como $\Sigma\left(n_{i} / t_{i}\right)$, donde $n_{i}$ es el número de plántulas germinadas durante el intevalo $t$, que es el tiempo desde la siembra hasta el día de conteo (ISTA, 2004).

\section{Análisis estadístico}

Se aplicó la transformación de la raíz cuadrada del arco seno en las variables que se registraron en porcentajes, antes de efectuar sus análisis de varianza. Estos análisis fueron con un diseño experimental completamente al azar en arreglo factorial $3 \times 5 \times 2$, correspondiente a tres tipos de chile, cinco etapas de desarrollo del fruto, y dos tiempos de extracción. Las comparaciones de medias se hicieron con la prueba de Tukey $(\mathrm{P}=0.05)$. Los análisis estadísticos se efectuaron con el paquete Statistical Analysis System versión 9.0 (SAS Institute, 2002).

\section{RESULTADOS Y DISCUSIÓN}

\section{Contenido de humedad}

La floración ocurrió a los $45 \mathrm{~d}$ después del trasplante (ddt) en los chiles Ancho y Guajillo, y a los 54 ddt en el chile de Árbol. A pesar de estas diferencias varietales en precocidad, las semillas de los tres tipos de chile cosechadas en todas las etapas tuvieron en promedio un contenido de humedad de $7.6 \%$ al momento de las pruebas, lo cual significa que todas las semillas pasaron por un proceso de desecación similar hasta alcanzar el equilibrio con el ambiente del sitio de almacenamiento $\left(18 \pm 2{ }^{\circ} \mathrm{C}\right)$. Este porcentaje fue considerado bajo y que no interfirió en el desempeño de las otras variables.

\section{Respuesta a tipos de chile y etapas de desarrollo}

Las fuentes de variación resultaron significativas en todas las variables, excepto el efecto de la extracción (E) sobre el peso volumétrico (PV) (Cuadro 1). Esto último indica que las etapas de extracción no indujeron cambios en el peso volumétrico de las semillas; es decir, que el PV fue el mismo, ya sea que hayan sido extraídas inmediatamente después de cosechar el fruto o después de haberlas dejado secar por 15 d. Los resultados del análisis de varianza también muestran el efecto del factor etapa de desarrollo (D) sobre las variables peso volumétrico, germinación y velocidad de germinación; en el peso de 1000 semillas el efecto de dicho factor fue superado por su interacción con el tipo de chile ( $\mathrm{T} x$ D), como se demuestra al comparar el valor relativo de los cuadrados medios de cada fuente de variación (Cuadro 1).

La evolución de las variables de respuesta para cada tipo de chile sigue de manera general un comportamiento sigmoidal, es decir, una fase de crecimiento inicial lento o nulo, seguida de una etapa de crecimiento exponencial y luego una atapa de desaceleración que culmina en un tamaño máximo en las últimas dos o tres etapas de desarrollo (Figura 1). Durante las tres etapas del desarrollo de la semilla señaladas por Taiz y Zeiger (2010), su calidad evoluciona hasta alcanzar un punto máximo en la madurez fisiológica, y después de ésta es que inicia el deterioro. Con los resultados de este estudio se puede inferir que al no 
Cuadro 1. Cuadrados medios y nivel de significancia estadística para las variables de calidad física y fisiológica de semillas de tres tipos de chile provenientes de frutos cosechados en cinco etapas de desarrollo y luego extraídas en dos periodos postcosecha.

\begin{tabular}{|c|c|c|c|c|c|}
\hline Fuentes de variación & Grados de libertad & PS (g) & $\mathrm{PV}\left(\mathrm{kg} \mathrm{hL}^{-1}\right)$ & PG (\%) & VG (radículas d $\left.\mathrm{d}^{-1}\right)$ \\
\hline Tipo (T) & 2 & $106.35^{* *}$ & $137.42^{* *}$ & $994.29^{* *}$ & $2.07^{* *}$ \\
\hline Etapa (D) & 4 & $470.00^{* *}$ & $2255.97^{* *}$ & $27,161.15^{* *}$ & $34.84^{* *}$ \\
\hline $\mathrm{T} \times \mathrm{D}$ & 8 & $2190.48^{* *}$ & $699.57^{* *}$ & $8480.51^{* *}$ & $10.97^{* *}$ \\
\hline Extracción (E) & 1 & $4.26^{* *}$ & 9.08 & $12,568.68 * *$ & $25.85^{* *}$ \\
\hline $\mathrm{TxE}$ & 2 & $218.80^{* *}$ & $59.89^{* *}$ & $3252.58 * *$ & $6.58^{* *}$ \\
\hline $\mathrm{D} \times \mathrm{E}$ & 4 & $210.17 * *$ & $1017.79^{* *}$ & $14,976.75 * *$ & $21.29^{* *}$ \\
\hline $\mathrm{T} \times \mathrm{D} \times \mathrm{E}$ & 8 & $76.11^{* *}$ & $343.94^{\star *}$ & $5165.30^{* *}$ & $7.35^{* *}$ \\
\hline Error & 90 & 0.04 & 2.18 & 45.64 & 0.07 \\
\hline $\mathrm{CV}(\%)$ & & 3.00 & 4.00 & 17.00 & 19.00 \\
\hline $\mathrm{R}^{2}$ & & 0.99 & 0.99 & 0.97 & 0.97 \\
\hline
\end{tabular}

** Significativo a P < 0.01; PS = peso de 1000 semillas; PV = peso volumétrico; PG = porcentaje de germinación; VG = velocidad de germinación.

existir germinación en las etapas E1 (25 dda) y E2 (40 dda) las semillas se encontrarían en la etapa de histodiferenciación, donde aún no alcanzaban el desarrollo morfológico y fisiológico que permitiera una germinación adecuada (Otho et al., 2007). En este periodo igualmente, las semillas mostraron un peso de 1000 semillas y peso volumético considerablemente bajos. Conforme avanzó más su desarrollo el valor de las cuatro variables evaluadas se incrementó hasta alcanzar un máximo. Estos datos demuestran que la etapa de desarrollo (D) a la cosecha de fruto es la principal fuente de variación en las variables evaluadas.

En chile Árbol, las semillas cosechadas a los 40 dda (etapa E2) se encontraban quizá al inicio de la etapa de acumulación de reservas, como propusieron Otho et al. (2007) pues apenas $17.6 \%$ germinaron, lo que permite inferir que en estas semillas el embrión contaba ya con las estructuras necesarias para germinar, pero no habían completado su madurez fisiológica y no contaban con las reservas suficientes para abastecer el crecimiento del embrión durante la germinación.

En los tres tipos de chile, la calidad fisiológica (PG y VG) alcanzó su máximo cuando las semillas se cosecharon durante la madurez del fruto (E4), con una sola excepción (Figura 1). La máxima calidad en chiles Ancho y Guajillo se obtuvo en esta etapa y se mantuvo durante E5 sólo para Guajillo, mientras que en chile de Árbol la máxima se alcanzó hasta E5 (Figura 1). Resultados similares fueron reportados por Sayed y Essam (1952), Edwards y Sundstrom (1987) y Valdés et al. (1992) en chile Bell.

La etapa de maduración de la semilla es una característica que está positivamente correlacionada con el vigor, definido éste como la suma total de las propiedades de la semilla que determinan el nivel potencial de actividad y desarrollo durante la germinación y emergencia de la plántula en condiciones adversas (ISTA, 2004). Así, las semillas extraídas en las etapa de desarrollo 4 y 5 fueron las más vigorosas, con excepción del chile Ancho que requirió llegar a la etapa 5 para alcanzar el máximo vigor; mientras que en las otras dos variedades tanto la variable VG como la germinación disminuyeron en 30 y $20 \%$ respectivamente, al llegar a la E5 (Figura 1). Esta disminución pudiera atribuirse a daños por la exposición a bajas temperaturas al interior del invernadero, causadas por una helada ocurrida durante esta etapa. Los otros tipos no presentaron cambios en estas variables e incluso las semillas de chile de Árbol, como ya se mencionó, presentaron la mayor calidad hasta la E5. Por lo anterior, es probable que este tipo de chile sea más resistente a este factor adverso.

El efecto de la interacción entre los tipos de chile y la fase de desarrollo fue el factor más importante en el comportamiento del peso de 1000 semillas (Cuadro 1), el cual aumentó rápidamente hasta la etapa $\mathrm{E} 3$ en los tres tipos de chile (Figura 1). La principal diferencia entre variedades fue el valor que alcanzaron en la fase final E5, cuando la semilla de chile de Árbol pesó $30 \%$ menos que la semilla de los otros dos tipos de chile.

\section{Respuesta a etapas de extracción e interacciones}

La interacción entre la etapa de desarrollo y el tiempo de extracción ( $\mathrm{D} \times \mathrm{E}$ ), cuyo efecto fue el segundo más importante segun los cuadrados medios (Cuadro 1), muestra que las semillas extraídas del fruto $15 \mathrm{~d}$ después de la cosecha (EP) superaron a la extracción efectuada en la cosecha (EI) 

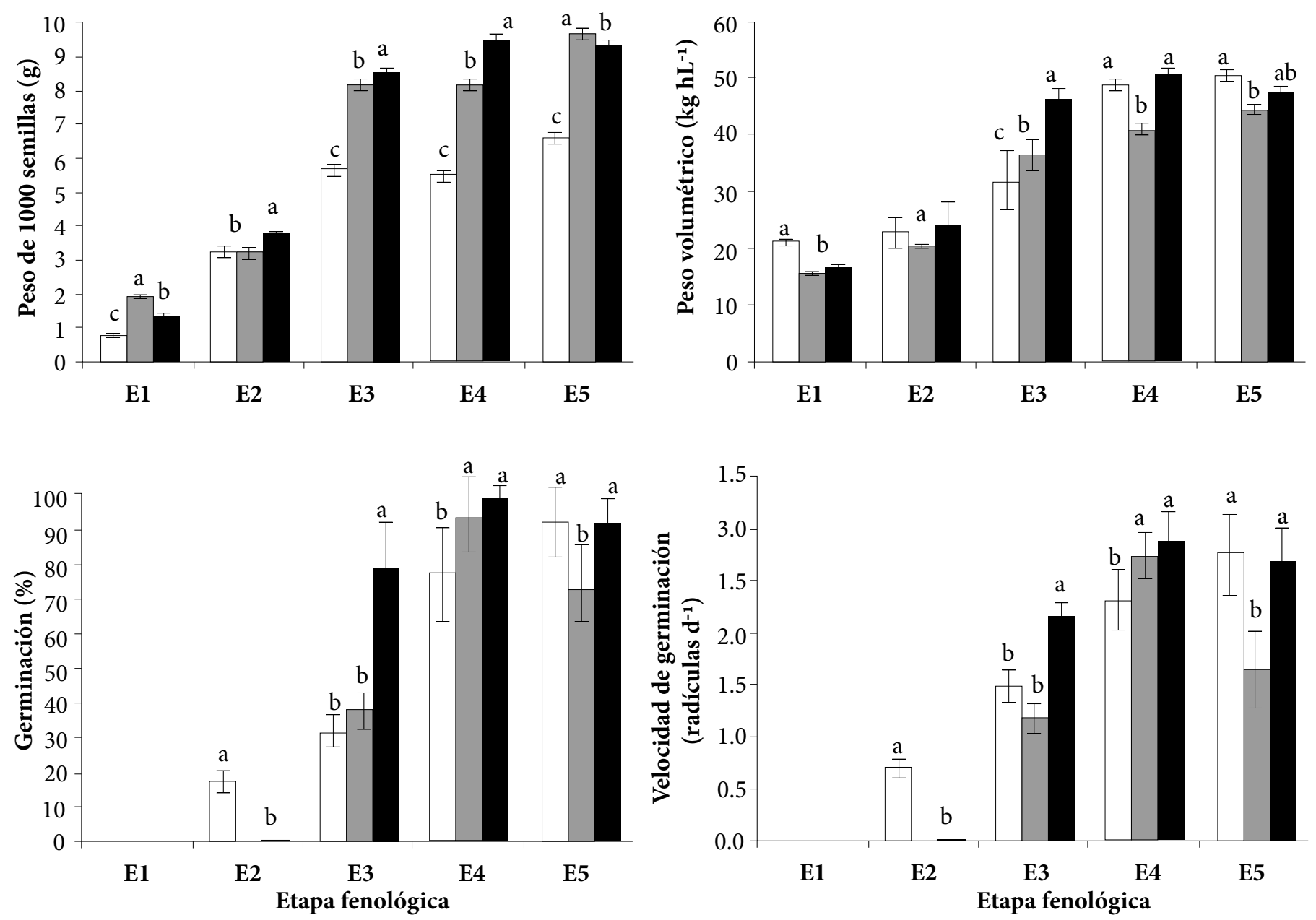

Figura 1. Evolución de la calidad de semillas de tres tipos de chile (de Árbol $\rightarrow$, Ancho $\rightarrow-$, y Guajillo $\rightarrow-$ ) provenientes de frutos cosechados en cinco etapas de desarrollo, en promedio de dos etapas de extracción poscosecha. E1 = 25 días después de antesis (dda); E2 = 40 dda; E3 = Fruto con $50 \%$ de cambio de color; E4 = Cambio total del color del fruto; E5 = Fruto seco. Medias con letras iguales no son estadísticamente diferentes (Tukey, 0.05).

en las variables PG y VG y en algunos casos también de PS y PV (Figura 2), siempre y cuando el embrión haya alcanzado un desarrollo completo, ya que en la etapa E1 (frutos de $25 \mathrm{~d}$ de edad) el embrión aún estaba inmaduro. En las semillas recién extraídas (EI) hasta la etapa E3 (frutos con $50 \%$ de color), la baja germinación (Figura 2) se atribuye a que las semillas aún no adquirían tolerancia a la deshidratación, característica que favorece el mantenimiento de la longevidad durante el almacenamiento (Otho et al., 2007). En las semillas extraídas después de permanecer $15 \mathrm{~d}$ dentro del fruto (EP) cosechado en E3, la germinación subió a más de $85 \%$, lo que indica que en este periodo adquieren la tolerancia a la desecación.

Los resultados obtenidos muestran que, de manera general, la interacción de las etapas de desarrollo con los tiem- pos de extracción fue significativa durante las cosechas en E2 y E3, y nula en E4 y E5, para las variables peso de 1000 semillas y peso volumétrico. Esto sugiere que cuando una semilla aún no ha acumulado su máxima biomasa es porque todavía recibe asimilados de otros tejidos del fruto, aun cuando éste ya había sido cosechado (Figura 2).

En el presente estudio se demuestra que la mayor calidad fisiológica de la semilla de estos chiles se obtiene cuando se extrae la semilla de frutos maduros (E3) y almacenados por $15 \mathrm{~d}$ (extracción posterior, EP). No obstante, a partir de la etapa de inicio de cambio de color del fruto con extracción posterior (EP) es posible obtener semillas vigorosas y con germinación uniforme, que podrían usarse es programas de mejoramiento genético con ciclos de selección más cortos, y también servirían para la producción de 

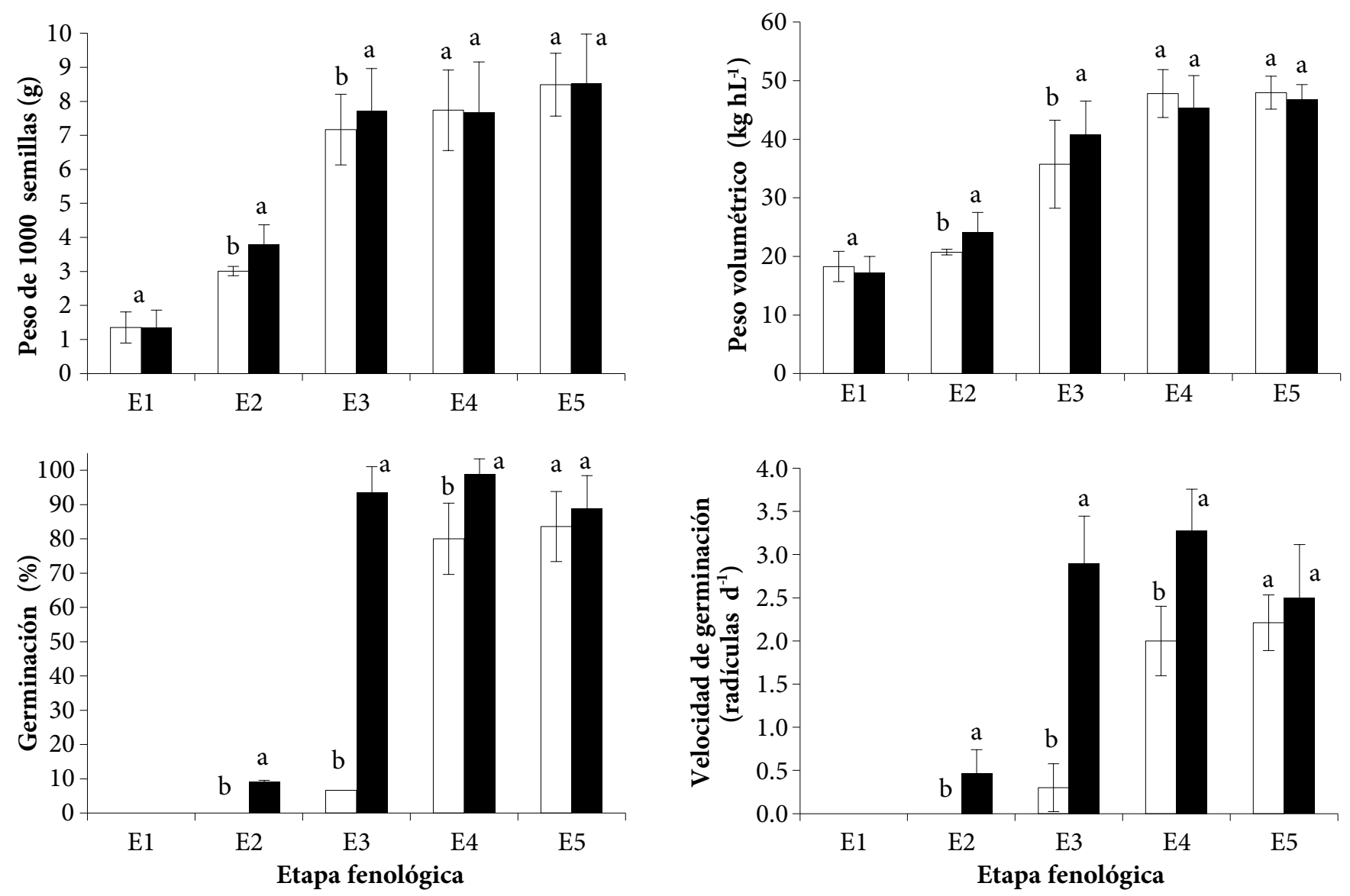

Figura 2. Evolución de la calidad de semillas de chile provenientes de frutos cosechados en cinco etapas de desarrollo, las cuales fueron extraídas inmediatamente después de la cosecha (EI, ) y después de $15 \mathrm{~d}$ dentro del fruto (EP, ), en promedio de tres variedades de chile. $\mathrm{E} 1=\mathbf{2 5}$ d después de antesis $(\mathrm{dda}) ; \mathrm{E} 2=40 \mathrm{dda}$; $\mathrm{E} 3$ = fruto con $50 \%$ de cambio de color; $\mathrm{E4}=$ cambio total del color del fruto; E5 = fruto seco. Medias con letras iguales no son estadísticamente diferentes (Tukey, 0.05).

semilla comercial pues son de suficiente calidad germinativa y se cosecharían en menos tiempo.

Entre las dos etapas de extracción, EI e EP, la mejor germinación obtenida en la mayoría de los tratamientos fue cuando se hizo la extracción posterior (EP). Por ejemplo, en frutos cosechados en E3 la extracción posterior mejoró la germinación en más de $100 \%$, porque la madurez de la semilla en algunas plantas está ligada a la senescencia del fruto. Es posible que al extraer las semillas sin haber avanzado en dicha senescencia, los procesos fisiológicos de maduración de la semilla sean incompletos (Bradford, 2004).

Tal vez la permanencia de las semillas dentro del fruto por $15 \mathrm{~d}$ después de haber sido cosechados permite la oclusión normal de los tejidos conectivos con el resto del fruto, sin dañar las cualidades de las semillas. Al respecto, Vidigal et al. (2009) reportaron que la conductividad eléctrica de las semillas disminuye durante el proceso de maduración de los frutos, lo que indica que está asociada con el desarrollo de una buena organización de la membrana celular, característica que favorece a la germinación. Así mismo, el proceso de adquisición de la tolerancia a la desecación que causa la pérdida de humedad de la semilla favorece la germinación, como se observó en tomate de cáscara, Physalis ixocarpa (Pérez-Camacho et al., 2008), y además la desecación promueve la degradación de hormonas inhibidoras de la germinación y el desarrollo de azúcares y proteínas protectoras de la membrana celular (Bewley et al., 2013).

\section{Cinética de la germinación}

La importancia de conocer la cinética de la germinación radica en que muestra el posible potencial de vigor de una semilla para generar una plántula vigorosa, por lo que una germinación lenta y con amplia dispersión en el tiempo 
produce plántulas heterogéneas y pequeñas (Matthews y Khajeh-Hosseini, 2006). En esta investigación la cinética de la germinación fue específica para cada tipo de chile, etapa de desarrollo y tiempos de extracción (Figura 3). En los chiles de Árbol y Ancho la germinación se inició a los $3 \mathrm{~d}$ después de haber instalado la prueba y en el chile Guajillo a los $4 \mathrm{~d}$ (Figura 3 ).

Las semillas extraídas inmediatamente (EI) germinaron en promedio $4 \mathrm{~d}$ después que las que tuvieron un reposo de $15 \mathrm{~d}$ dentro del fruto (EP) y con valores menores de germinación total acumulada. Las semillas obtenidas de frutos cosechados en las etapas E1 en ambos tiempos de extracción y de frutos en E2 con extracción inmediata (E2 EI) fueron incapaces de germinar, seguramente por estar aún inmaduras y contener cantidades importantes de inhibidores de la germinación, ya que estos inhibidores disminuyen conforme la semilla alcanza la madurez (Trigiano et al., 2008). La germinación acumulada total en el chile de Árbol del tratamiento E2 (etapa de desarrollo muy temprana) con extracción posterior alcanzó un valor relativamente alto (58 $\%)$ aunque haya iniciado la germinación $4 \mathrm{~d}$ después que la del tratamiento más vigoroso (E4 EP).

Esto sugiriere que dicho retraso pudo deberse a la lenta reactivación de las reservas acumuladas hasta esa etapa, o a que requirió esperar a que se lavaran sustancias inhibidoras de la germinación con el agua de riego. Aunado a lo anterior, el reposo de los frutos durante $15 \mathrm{~d}$ pudo coadyuvar a la desactivación o eliminación de sustancias inhibidoras.

En el chile Guajillo la semilla extraída inmediatamente de frutos cosechados en la fase E3 (E3 EI) alcanzó hasta 44 $\%$ de germinación a los $17 \mathrm{~d}$ de iniciado el proceso, lo que contrasta con los otros tipos de chile en los que con la misma combinación de tratamientos se obtuvieron valores de germinación cercanos a cero. Así mismo, en chile de Árbol el tratamiento de cosecha de fruto maduro y con extracción inmediata de semilla (E4 EI) tuvo una germinación de $52 \%$, que fue inferior a la del tratamiento de fruto cosechado al inició de cambio de color y con extracción posterior (E3 EP). Estos resultados demostrarían, junto con los de E2 EP, la importancia en chile de Árbol de hacer la extracción posterior de semilla de frutos cosechados en etapas tempranas de desarrollo. En estudios futuros convendría ampliar los periodos de extracción para medir su impacto en la germinación.

En los chiles de Árbol y Ancho el tratamiento E4 EP, y en Guajillo el tratamiento E3 EP, produjeron la germinación más rápida, con valores de 93, 99 y $98 \%$, respectivamente. En los tres tipos de chile la máxima velocidad de germinación ocurrió entre los 6 y los 11 d, independientemente de la combinación de tratamientos. De acuerdo con las recomendaciones de la ISTA (2004), el conteo final de una prueba de germinación estándar debe hacerse a los $14 \mathrm{~d}$ después de la siembra (dds); en esta investigación, en el conteo efectuado ese día se obtuvo el mayor porcentaje de germinación de las semillas consideradas más vigorosas, que corresponden a la que se extrajeron $15 \mathrm{~d}$ después de la cosecha de frutos totalmente maduros (EP).

Sin embargo, en algunos lotes de semillas, especialmente los del chile Ancho (E3 EP, E4 EI, E5 EI y E5 EP), todavía se detectó aumento de la germinación después de los 14 dds, lo que indicaría que para evaluaciones posteriores podrían considerarse conteos hasta los $18 \mathrm{dds}$, si se buscara obtener las plántulas homogéneas, pues en lotes de semilla de chile Pimiento se obtuvieron plántulas de mayor tamaño y menos variables cuando provinieron de semillas que germinaron en un menor tiempo (Demir et al., 2008).

\section{CONCLUSIONES}

La etapa de desarrollo del fruto fue el factor que más influyó en la calidad fisiológica de la semilla (germinación y vigor) de los chiles estudiados, ya que alcanzaron su valor máximo cuando fueron extraídas de frutos cosechados en las etapas de cambio de color (Ancho y Guajillo) y fruto seco (de Árbol). El peso de 1000 semillas aumentó rápidamente hasta la fase de inicio de color del fruto en los tres tipos de chile, y la semilla de chile de Árbol fue en promedio $30 \%$ menos pesada que la de los otros dos chiles. La extracción de la semilla $15 \mathrm{~d}$ después de la cosecha del fruto en etapas tempranas del desarrollo mejora significativamente la calidad física (peso de 1000 semillas y peso volumétrico) y la calidad fisiológica, ya que en la etapa de inicio de cambio de color la germinación alcanzó un promedio de 93.4 $\%$, muy superior a obtenida con las semillas de extracción inmediata (6.7\%).

La mejor calidad fisiológica observada en la cinética de germinación de semillas de los chiles Ancho y Árbol se alcanzó al extraerlas $15 \mathrm{~d}$ después de la cosecha de frutos completamente rojos, mientras que en Guajillo se logró el máximo cuando los frutos se cosecharos al inicio de cambio de color y las semillas se extrajeron $15 \mathrm{~d}$ después, por lo que se consideran las mejores etapas para cosechar la semilla.

\section{BIBLIOGRAFÍA}

Aguilar-Rincón V. H., T. Corona-Torres, P. López-López, L. Latournerie Moreno, M. Ramírez-Meraz, H. Villalon-Mendoza y J. A. Aguilar-Castillo (2010) Los Chiles de México y su Distribución. SINAREFI, Colegio de Postgraduados, INIFAP, IT-Conkal, UANL, UAN. Texcoco, Estado de México, México. $114 \mathrm{p}$.

Bewley J. D., K. J. Bradford, W. M. H Hilhorst and H. Nonogaky (2013) Seed. Physiology of development, germination and dormarcy. Third Edition. Springer. New York, U.S.A. 392 p.

Bradford K. J. (2004) Seed Production and Quality. Department of Vegetable Crops. University of California. Davis, California, USA. 


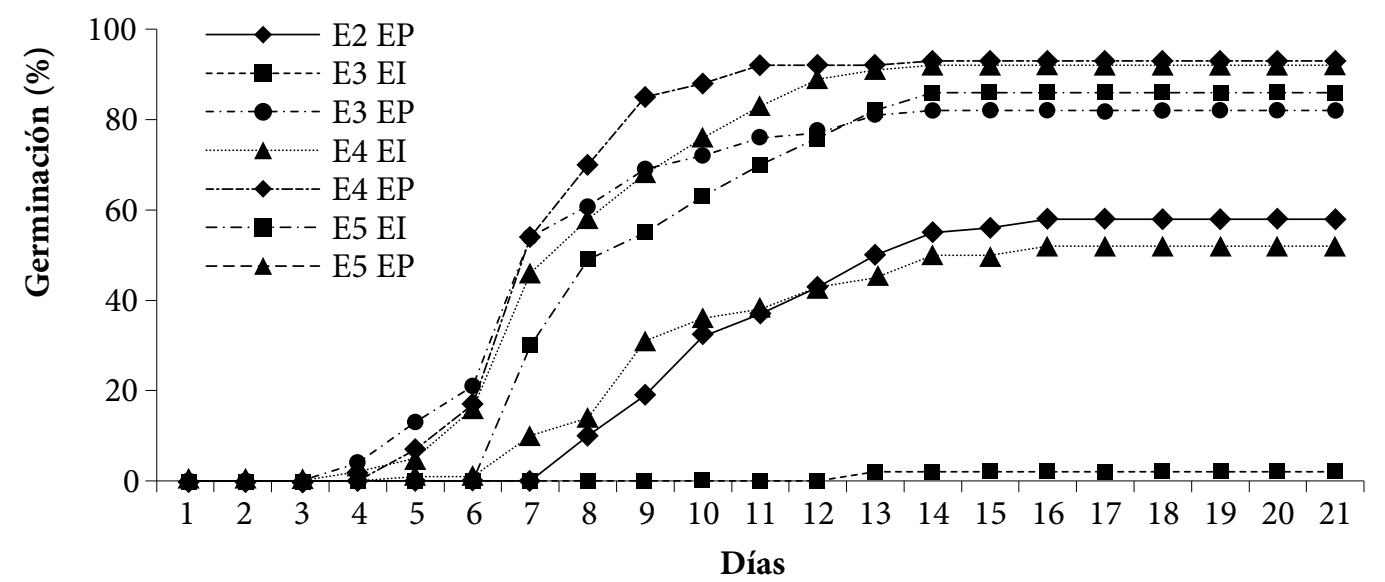

b
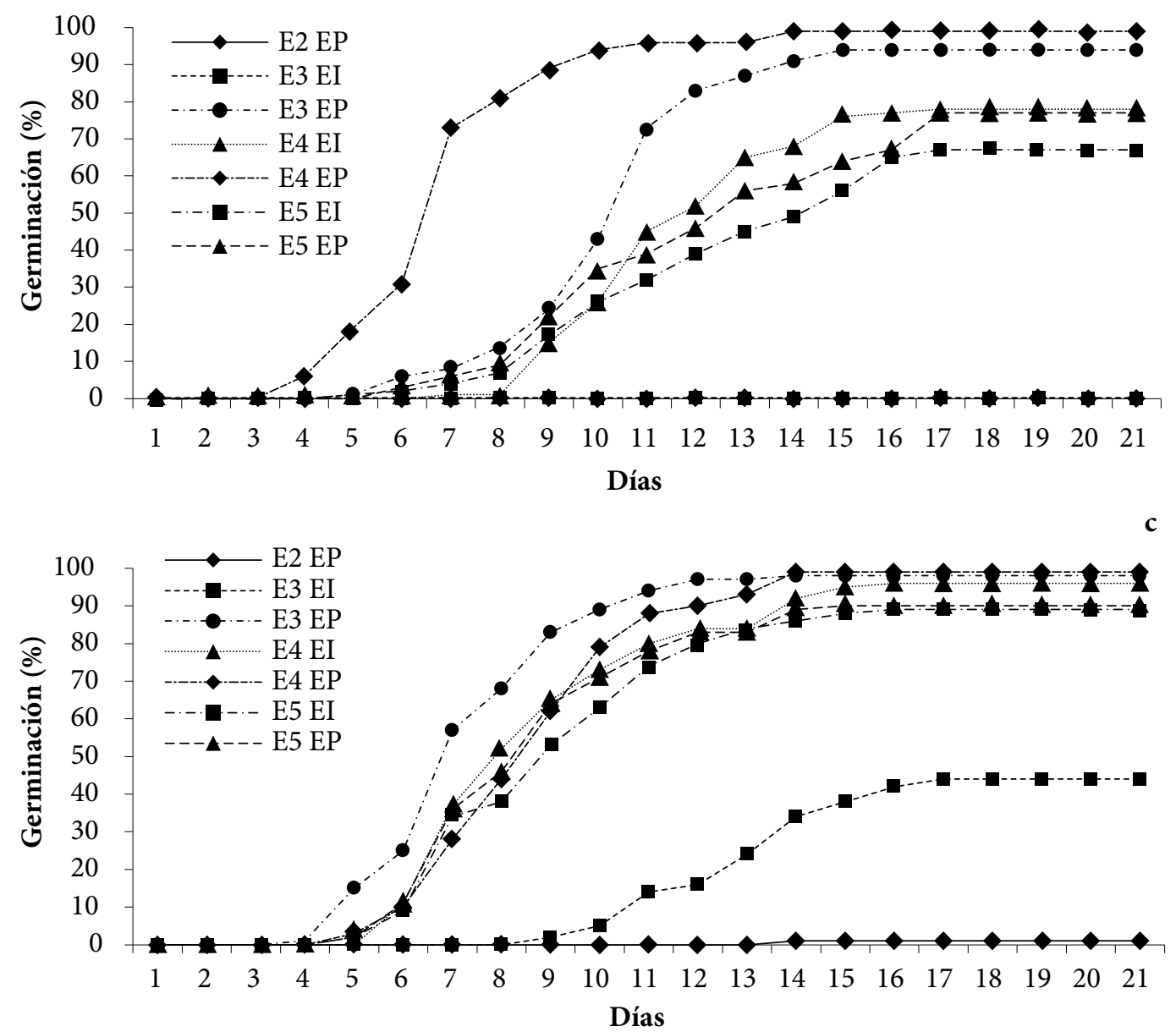

Figura 3. Germinación acumulada de semillas de chile de Árbol (a), Ancho (b) y Guajillo (c). Las semillas fueron obtenidas de frutos en: $\mathrm{E} 2$ = 40 d después de antesis; E3 = fruto con 50 \% de cambio de color; E4 = cambio total del color del fruto; E5 = fruto seco; semillas extraídas en la cosecha $(\mathrm{EI})$ y después de $15 \mathrm{~d}(\mathrm{EP})$. El resto de tratamientos no presentó germinación. 
$134 \mathrm{p}$.

Demir I., S. Ermis, K. Mavi and S. Matthews (2008) Mean germination time of pepper seed lots (Capsicum annuum L.) predicts size and uniformity of seedlings in germination tests and transplant modules. Seed Science and Technology 36:21-30.

Edwards R. L. and F. J. Sundstrom (1987) Afterripening and harvesting effects on Tabasco pepper seed germination performance. HortScience 22:473-475.

ISTA (International Seed Testing Association) (2004) International Rules for Seed Testing. Rules 2004. ISTA Editions. Bassersdorf, CH-Switzerland. $243 \mathrm{p}$.

Long-Solís J. (1986) Capsicum y su Cultura. La Historia del Chilli. Fondo de Cultura Económica. México, D.F. México. 181 p.

Matthews S. and M. Khajeh-Hosseini (2006) Mean germination time as indicator of emergence performance in soil of seed lots of maize (Zea mays). Seed Science and Technology 34:339-347.

Nuez F., R. Gil Ortega and J. Costa (2003) El Cultivo de Pimientos, Chiles y Ajíes. Ed. Mundi-Prensa. Madrid, España. 586 p.

Otho M. A., S. L. Stone and J. J. Harada (2007) Genetic control of seed development and seed mass. In: Seed Development, Dormancy and Germination. K J Bradford, H Nonogaki (eds). Blackwell Publishing. Iowa, USA. pp:1-49.

Pérez-Camacho I., V. A. González-Hernández, J. C. Molina-Moreno, 0. J. Ayala-Garay y A. Peña-Lomelí (2008) Efecto de desarrollo y secado de semillas de Physalis ixocarpa Brot. en germinación, vigor y contenido de azúcares. Interciencia 33:762-766.

Pittcock J. K. (2008) Seed production, processing and analysis. In: Plant
Propagation. C A Beyl, R N Trigiano (ed.). CRC Press Taylor \& Francis Group. U.S.A. pp:401-406.

Randle W. M. and S. Honma (1981) Dormancy in peppers. Scientia Horticulturae 14:19-25.

SAS Institute (2002) User's Guide of SAS (Statistical Analysis Sytem) Version 9. SAS Intitute. 9th ed. Cary, North Carolina, USA. 550 p.

Sayed M. S. and M. Essam (1952) Viability of seeds harvested from fruits at different stages of maturity. Proceedings of American Society for Horticultural Science 60:327-329.

SIAP, Servicio de Información Agroalimentaria y Pesquera (2011) Anuario del Servicio de Información Agroalimentaria y Pesquera, ciclos 2010. Secretaría de Agricultura, Ganadería, Desarrollo Rural, Pesca y Alimentación. Disponible en: http://www. siap.gob.mx (Abril 2011).

Taiz L. and E. Zeiger (2010) Plant Physiology. 5th ed. Sinauer Associates, Inc., Publishers. Sunderland, Massachusetts, USA. 764 p.

Trigiano R. N., R. A. Follum and C. A. Beyl (2008) Sexual reproduction in angiosperms. In: Plant Propagation. C A Beyl, R N Trigiano (eds.). CRC Press Taylor \& Francis Group. US.A. pp:379-389.

Valdés V., P. Anguita y C. Ulriksen (1992) Efecto del estado de madurez de los frutos de pimiento (Capsicum annuum L.) sobre la calidad de la semilla [Keystone Giant Resistant, Maor]. Ciencia e Investigación Agraria 19:3-7.

Vidigal D. S., D. C. F. S. Dias, E. R. V. Von Phino and L. A. S. Dias (2009) Sweet pepper seed quality and lea-protein activity in relation to fruit maturation and post-harvest storage. Seed Science and Technology 37:192-201. 